\title{
LAS TRADICIONES CENTRO-ANDINAS DE ROCAS GRABADAS (PERÚ): EVOLUCIONES Y CONTINUIDADES*
}

\author{
CENTRAL-ANDEAN ENGRAVED ROCK TRADITIONS (PERU): \\ EVOLUTION AND CONTINUITIES
}

\author{
Jean Guffroy ${ }^{1}$
}

\begin{abstract}
El análisis de diversos criterios referentes a la ubicación y a las características de varios conjuntos de rocas grabadas permite precisar las evoluciones ocurridas, tanto en la organización de los sitios como en los estilos e iconografías asociadas, de todo el Perú. Es también posible reconocer singularidades regionales que reflejan probablemente usos y funciones particulares. Por otro lado, existen diferentes rasgos que se repiten sobre un vasto territorio y un importante lapso de tiempo, indicando continuidades en estas manifestaciones rupestres relacionadas con actividades sociales y religiosas probablemente múltiples.
\end{abstract}

Palabras claves: arte rupestre, petroglifos, iconografía andina, rituales precolombinos.

By analyzing various criteria concerning the situation and characteristics of engraved rocks from several localities, the evolution that occurred in site organization, styles, and in related iconographies in all of Peru can be clarified. Regional singularities, which probably reflect specific uses and functions, can also be ascertained. Additionally, various features have been found repeatedly within a large territory over a long time span, indicating continuities in these artistic expressions, related to multiple social and religious activities.

Key words: Rock art, petroglyphs, Andean iconography, Precolumbian rituals.

El análisis de los contextos en los cuales se encuentran las piedras grabadas, de la organización de los sitios, así como de los estilos y temas tratados, han permitido definir la existencia de diversas tradiciones marcadas por una cierta evolución en los usos y funciones del arte rupestre del Perú (Guffroy 1999, 2003, 2007). Aunque existen, en el Sur del país, unas escasas muestras de arte grabado que pueden ser asociadas a grupos de cazadores recolectores (Bustinza 1991), esta modalidad rupestre parece estar ligada, ante todo, al desarrollo de las sociedades agroalfareras. A las figuras mitológicas frecuentemente aisladas del largo periodo Formativo (Tradición A: 2.500-300 a.C.), principalmente presentes en la costa norte del Perú, suceden las representaciones agrupadas de seres humanos o sobrenaturales, pequeños animales, signos geométricos, figuras complejas y cúpulas (hoyuelos circulares poco profundos) del período Intermedio Temprano (300 a.C-600 d.C.), con una difusión hacia el sur y norte de la primera zona (Tradición B). Una tercera gran Tradición C, caracterizada por representaciones más esquematizadas y estereotipadas de hombres y animales, aparece más tardíamente (entre el Horizonte Medio y el periodo Inca) (600-1.532 d.C.) en el sur del Perú, donde la técnica del grabado sobrevive hasta después de la conquista.

A pesar de estas importantes evoluciones, existen indicios, de diversas naturalezas, que permiten establecer lazos entre sitios dispersos en el espacio y el tiempo (Figura 1). La repetición de ciertas asociaciones, tal como la presencia de caminos o de sectores de producción, sugieren que estamos frente a un mismo fenómeno cultural, asociado a prácticas rituales y sociales que evolucionaron durante los tres últimos milenios de desarrollo de las sociedades agrarias precolombinas del Perú.

Una revisión detallada de diferentes criterios permite establecer la existencia, dentro de las grandes tradiciones antes mencionadas, de complejos singulares -que reflejan mejor las evoluciones ocurridas-así como de particularismos regionales o culturales -probablemente ligados a la diversidad

\footnotetext{
* Este estudio fue presentado en el simposio sobre Arte Rupestre de los Andes, realizado en el $52^{\circ}$ Congreso Internacional de Americanistas, Sevilla, julio de 2006. La edición de los artículos seleccionados para este número de la Revista estuvo a cargo de Marcela Sepúlveda y Jean Guffroy.

1 Centre IRD-Orléans, 5 rue du carbone, 45072, Orléans, Cedex 02, France. jean.guffroy@ird.fr
} 


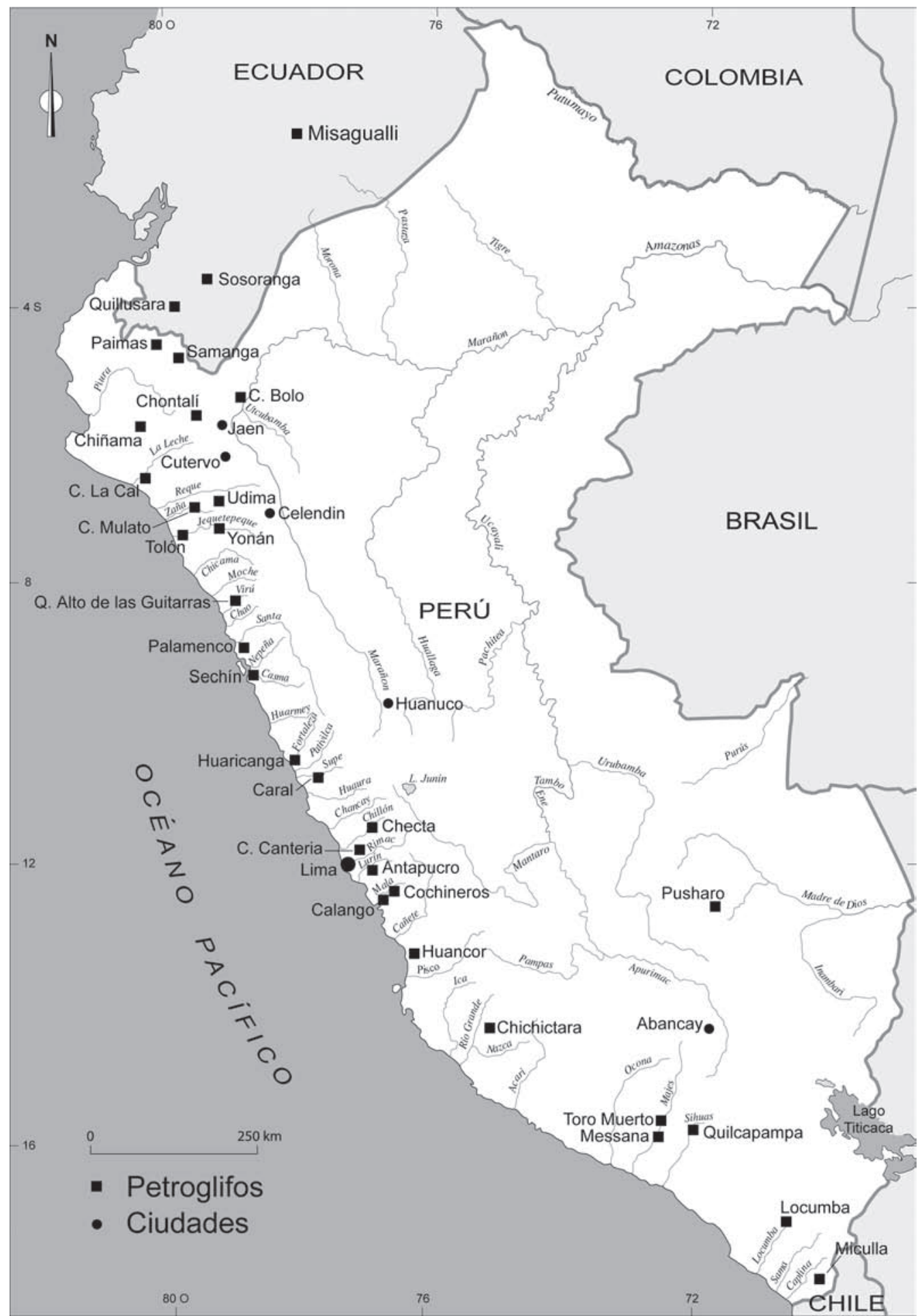

Figura 1. Emplazamiento de los principales sitios mencionados en el texto. Location of the main sites references in the text. 
de los rituales asociados a los sitios de petroglifos. Adicionalmente, se vislumbran también las continuidades y fundamentos que ligan cada tradición. Varios de estos datos significativos ya han sido expuestos en trabajos anteriores (Guffroy 1999, 2007), resumiéndolos al máximo en esta ocasión para insistir mayormente sobre las correlaciones que parecen más relevantes, y así llegar a una mejor comprensión de las finalidades y usos de las manifestaciones rupestres.

\section{La Cantidad de Piedras Grabadas}

El número de piedras grabadas en cada sitio constituye, sin duda, un primer elemento importante que refleja tanto las actividades ocurridas como su finalidad y su inscripción en el paisaje cultural local.

Las localidades que contienen un número reducido de piedras grabadas (entre una y diez) representan la mayoría de los yacimientos registrados, hasta ahora en el Perú, y muy probablemente existan muchos más sitios por descubrir. Su interpretación plantea diversos problemas y revela la existencia de diferentes casos y contextos.

La primera noción cuestionable es aquella del aislamiento real de una piedra o grupo de piedras; o en otras palabras, de continuidad y discontinuidad entre los conjuntos de grabados. Este aislamiento parece ser absoluto en varios sitios, pero muy relativo en otras oportunidades -tal como el caso de los sitios satélites, ubicados en un radio de unos kilómetros alrededor de un sitio importante, situación que se repite frecuentemente. Así, en Checta, existen, de ambos lados del sitio principal (con 430 piedras grabadas), otras cinco localidades situadas a proximidad contienen entre una y diez rocas con petroglifos. Todas pertenecen claramente a un mismo dispositivo y conjunto, y el aislamiento de algunas piedras es más aparente que real. La presencia de tales organizaciones espaciales parece bastante generalizada en la costa central, en otros sitios como Huancor y Chichictara, entre otros (Núñez Jiménez 1986).

En el sitio de Udima/Poro-Poro, en el valle de Zaña (Del Carpio et al. 2001), hay en dos localidades diferentes, una piedra con una representación compleja de un ser antropomorfo (un guerrero y un músico) de estilo Cuspinique tardío. Cada piedra parece aislada, pero existe en la misma zona: pinturas polícromas del mismo estilo y diversas estructuras ceremoniales formativas. De nuevo, este aislamiento es muy relativo; pues, todas estas manifestaciones forman, en realidad, parte de una misma apropiación cultural de un territorio.

Otra distribución significativa-que aparece en la costa y sierra baja del extremo norte del Perú, en el sur del Ecuador (González 2004), así como en las vertientes orientales cercanas- se refiere a la presencia de piedras aisladas, con características comunes, dispersas sobre un vasto territorio. En algunos casos, tales como en las regiones de Frias, Paimas y Ayabaca en Piura (Polia 1995) o el valle del rio Chunchuca, en Jaén (Gamonal 2006), las distancias entre las piedras pueden alcanzar varios kilómetros, mientras que en otros casos, como en Samanga (Ayabaca), varían entre algunas decenas o centenares de metros. Tal repartición aparece también en zonas orientales más norteñas (cuenca de los ríos Upano, Napo y Misagualli en Ecuador) y sureñas (La Convención en el Cusco).

Otra situación, recientemente caracterizada (Guffroy 2007) corresponde a la asociación recurrente de una piedra con cúpulas o con un petroglifo sencillo (espiral, antropomorfo) con restos arquitecturales tempranos del período Precerámico Final. En todos los casos, las piedras grabadas no se integran directamente a la arquitectura del sitio habiendo sido, sin dudas, dispuestas en el lugar después del abandono de las estructuras ceremoniales. Esta asociación singular aparece en los sitios de Caral (río Supe; Shady 2005), Huaricanga (río Fortaleza), Sechín (río Casma; Núñez Jiménez 1986) y Udima (río Zaña). Las piedras podrían haber sido puestas allí a manera de recordatorio o con fines votivos.

Las piedras realmente aisladas corresponden en su mayoría a bloques de grandes tamaños, visibles desde lejos. Se encuentran a menudo en las cercanías de los ríos y quebradas o en las partes altas de los cerros. Este aislamiento parece reflejar -en las zonas norteñas y orientales- un verdadero modelo de distribución, ligado con usos particulares, mientras que parece indicar en otros casos (ciertos valles de la costa central y sur) un desarrollo local limitado del arte grabado.

Los sitios que contienen entre diez y cincuenta piedras grabadas son bastante numerosos. En diversos valles costeros corresponden a la agrupación más importante de su cuenca. Los pequeños sitios predominan en la etapa temprana, es decir, durante el Formativo: Tolón, Cerro San Simón, Quebrada del Felino (Pimentel 1986), Cerro Cantería (Abanto 
y García-Godos 2004). Las figuras tempranas aparecen también en yacimientos (Samanga, Alto de las Guitarras, Palamenco, Chichictara; Nuñez Jiménez 1986) que contienen mayormente petroglifos más recientes. Representan, en estos casos, el inicio de una tradición seguida después durante varios siglos. Sin embargo, los sitios de muy larga duración o que representan atribuciones culturas sucesivas no son tan frecuentes, y la iconografía de cada sitio es, por lo general, bastante homogénea, correspondiente a un mismo y único lapso temporal.

Los sitios con más de cincuenta piedras grabadas no son muy numerosos, aunque se encuentran dispersos a todo lo largo de la vertiente andina occidental. La presencia de centenares de piedras es todavía menos frecuente, con apenas una docena de sitios para todo el Perú. Estas grandes concentraciones no existen en las zonas serranas norteñas y orientales. La acumulación de figuras grabadas implica el uso repetido del sitio durante un lapso de tiempo importante. Como ya lo notáramos previamente (Guffroy 2007), el acto de grabar no parece haber sido realizado durante largos períodos; las actividades realizadas durante y después del grabado tuvieron probablemente un rol clave, no siempre fácil de vislumbrar. Sin embargo, en varios de estos sitios (Alto de las Guitarras, Cerro Mulato, Yonán, Checta, Huancor, Toro Muerto) existen indicios como superposiciones, mutilaciones (van Hoek 2005), cambios iconográficos o presencia de diferentes patinas que indican la probable existencia de diferentes momentos de ejecución, reflejo de ciertas evoluciones ideológicas.

\section{La Distribución de las Piedras}

Otro criterio importante corresponde a la topografía de los sitios escogidos y a la distribución de las piedras grabadas.

Un tipo común, en todas las regiones y épocas, corresponde a los sitios de planicie. Las piedras están dispersas sobre una superficie plana o con ligera pendiente, cuya extensión puede variar entre menos de una y varias decenas de hectáreas. Desde este punto de vista, se pueden reconocer concentraciones densas (tal como Checta) o más difusas (Palamenco, Toro Muerto, Samanga), con piedras más aisladas en su entorno. El acto de grabar pudo ser en ciertos casos incómodo (implicando de estar echado al suelo, por ejemplo), pero nunca muy difícil. En estos yacimientos, el recorrido entre las piedras, así como el hecho de mirar los petroglifos, son acciones también fáciles. Por lo general, se requiere cierta proximidad a la roca para ver a las figuras grabadas, las que también suelen estar presentes sobre piedras de muy pequeño tamaño.

Otro tipo de sitio corresponde a los bloques ubicados sobre los pendientes o estribaciones de los cerros (Figura 2). Las rocas están a menudo acumuladas en cascada, y tanto el acto de grabar como de encontrar y mirar ciertos petroglifos son tareas incómodas, hasta peligrosas (Cerro Mulato, Yonán, Huancor). Ciertas figuras son de gran tamaño y se observan desde lejos, mientras otras se encuentran bastante escondidas. Estos yacimientos tienen un aspecto teatral bien marcado, a manera de anfiteatros.

Existen también petroglifos ubicados en quebradas, algunos sobre las paredes rocosas y otros sobre las piedras acumuladas al fondo. En algunos casos, tal como en Quilcapampa, el acto de grabar pudo ser peligroso y haber necesitado alguna ayuda técnica como el uso de soga o de andamio. Los petroglifos son invisibles cuando se entra al fondo de la quebrada y sólo se pueden apreciar desde arriba y de lejos. Estos sitios en quebradas son más frecuentes en el extremo sur peruano (Quilcapampa, Locumba, Quebrada Palka (Gordillo 1996), donde se encuentran asociados a sectores de inhumaciones bajo la modalidad de tumbas circulares.

En los grandes sitios, la distribución de ciertos tipos de piedra o de ciertos temas iconográficos sugiere también un cierto grado de organización. En Checta (Guffroy 1999) se nota claramente la existencia de un pequeño grupo de tres piedras aisladas de las demás -cubiertas con figuras similares y cúpulas alineadas- que parecen marcar la entrada. En la parte central del sitio existen dos otras rocas grandes y planas cubiertas con cúpulas. Éstas se encuentran un poco apartadas, mientras que otro bloque con cúpulas y petroglifos marca la parte superior del yacimiento. La existencia de piedras o temas asociados con el acceso (la entrada o la salida) de los sitios parece estar también presentes en yacimientos como Yonán, Palamenco o Huancor. La existencia de una organización ceremonial del espacio con grabados se manifiesta en estos grandes sitios, por la presencia de sectores con características singulares, probablemente dirigidos o usados para la realización de actividades particulares. 


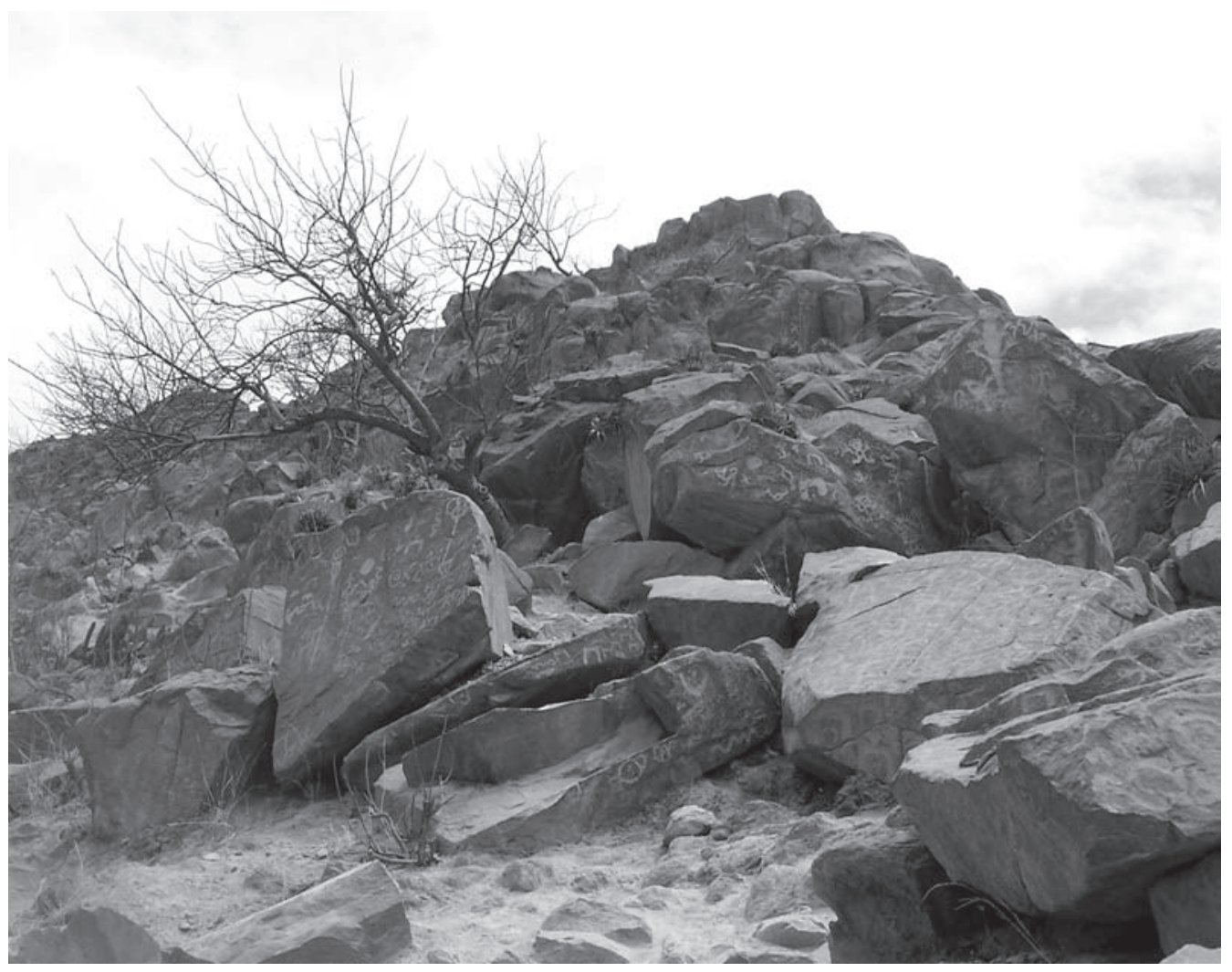

Figura 2. Petroglifos en un sitio de pendiente (Yonán, río Jequetepeque).

Petroglyphs from a sloped site (Yonán Jequetepeque River).

\section{Asociaciones Naturales y Culturales}

Ciertos elementos del paisaje pueden haber jugado un papel importante en la ubicación de las piedras grabadas. Algunas formaciones rocosas localizadas cerca de los grandes sitios semejan caras humanas vistas de perfil (Checta, Huancor) o de frente (Alto de las Guitarras) o presentan características geológicas singulares (Yonán, Messana/ Punta Colorada). La presencia de rocas cupríferas (Huancor), de fuentes de agua dulce (Toro Muerto) o salada (Alto de las Guitarras; Campana 2007) tuvo probablemente su importancia. Una posición dominante en cuanto al valle cultivable (Checta, Chichictara, Huancor, Toro Muerto), la presencia de una confluencia de ríos (Yonán, Locumba) o la cercanía de caminos de circulación constituyen otros rasgos repetidos cuya real significación es difícil determinar. Ya hemos insistido en diversas publicaciones (Guffroy 1999, 2007) sobre la relación privilegiada que pudo existir entre varios sitios de petroglifos y los sectores de cultivo de la coca durante la época prehispánica, en la costa norte y central del Perú. Tal asociación puede también ser significativa en regiones orientales más apartadas, tal como en la provincia de la Convención en Cusco (Valcarcel 1926), o en el sitio de Shanshipampa, en el extremo norte del Ecuador (Bray 2001).

Existen igualmente relaciones significativas entre las piedras grabadas y otras manifestaciones culturales. Señalamos, anteriormente, la asociación repetida de una piedra grabada con estructuras ceremoniales tempranas. En varios otros sitios se encuentran restos de pequeños recintos circulares o rectangulares, estelas paradas, piedras con pocitos que reflejan la existencia de diferentes rituales. Pequeños geoglifos están también presentes en sitios de petroglifos del sur del país (Toro Muerto, Locumba).

La presencia de inhumaciones cercanas a las piedras grabadas constituye un elemento muy interesante, particularmente difundido en los sitios sureños tardíos, en grandes sitios tales como Toro Muerto, Quilcapampa, Locumb y Miculla, pero 
también en un gran número de sitios de menor importancia (Hostnig 2003). Es difícil determinar la naturaleza de la relación y el grado de interacción de los dos fenómenos. En la primera descripción de Toro Muerto entregada por Linares (1960), este autor insiste en el hecho que el material funerario frecuentemente suele estar bajo cada petroglifo y acepta una probable contemporaneidad de las dos manifestaciones, sin establecer lazos más directos. La falta de excavaciones arqueológicas minuciosas no permite confirmar el carácter sistemático de tal asociación. En un sitio tal como la quebrada San Antonio en Locumba, la cantidad enorme de tumbas saqueadas frente al número relativamente reducido de piedras grabadas parece indicar un cierto predominio de la función funeraria, pero tampoco permite definir precisamente la relación cronológica o ideológica de las diversas manifestaciones. Existen, en otro sector del mismo sitio, rocas grabadas sobre una planicie, sin tumbas pero con recintos o geoglifos de piedras alineadas.

Dados los actuales avances de la investigación acerca del arte rupestre, no existe ninguna evidencia arqueológica que demuestre que el hecho de grabar estuvo directamente ligado al ritual funerario, así como que ocurra una exacta contemporaneidad entre el cavado de las tumbas y el grabado de las piedras. El testimonio de Duarte Fernández (Duviols 1971:57-58) -quien encontró, al principio del siglo XVII, entierros recientes alrededor de la piedra de Calango- indica que el entorno de esta roca seguía siendo utilizado como zona de entierros siglos después de su grabado, pese la ruptura cultural introducida por la conquista. Así, en varios sitios, la presencia de las piedras grabadas -otorgando al terreno un carácter sagrado- puede muy bien haber antecedido su uso como cementerio. Contrariamente, tampoco se puede descartar que, en otros casos, se hayan grabado petroglifos sobre rocas ubicadas dentro o cerca de un cementerio preexistente. No obstante, la repetición de esta asociación, así como la semejanza de las estructuras funerarias asociadas (pozos circulares delimitados por grandes bloques; Figura 3) dejan entrever una relación ritual bastante estrecha, difundida principalmente en la parte sureña del país, desde el Horizonte Medio hasta después de la conquista.

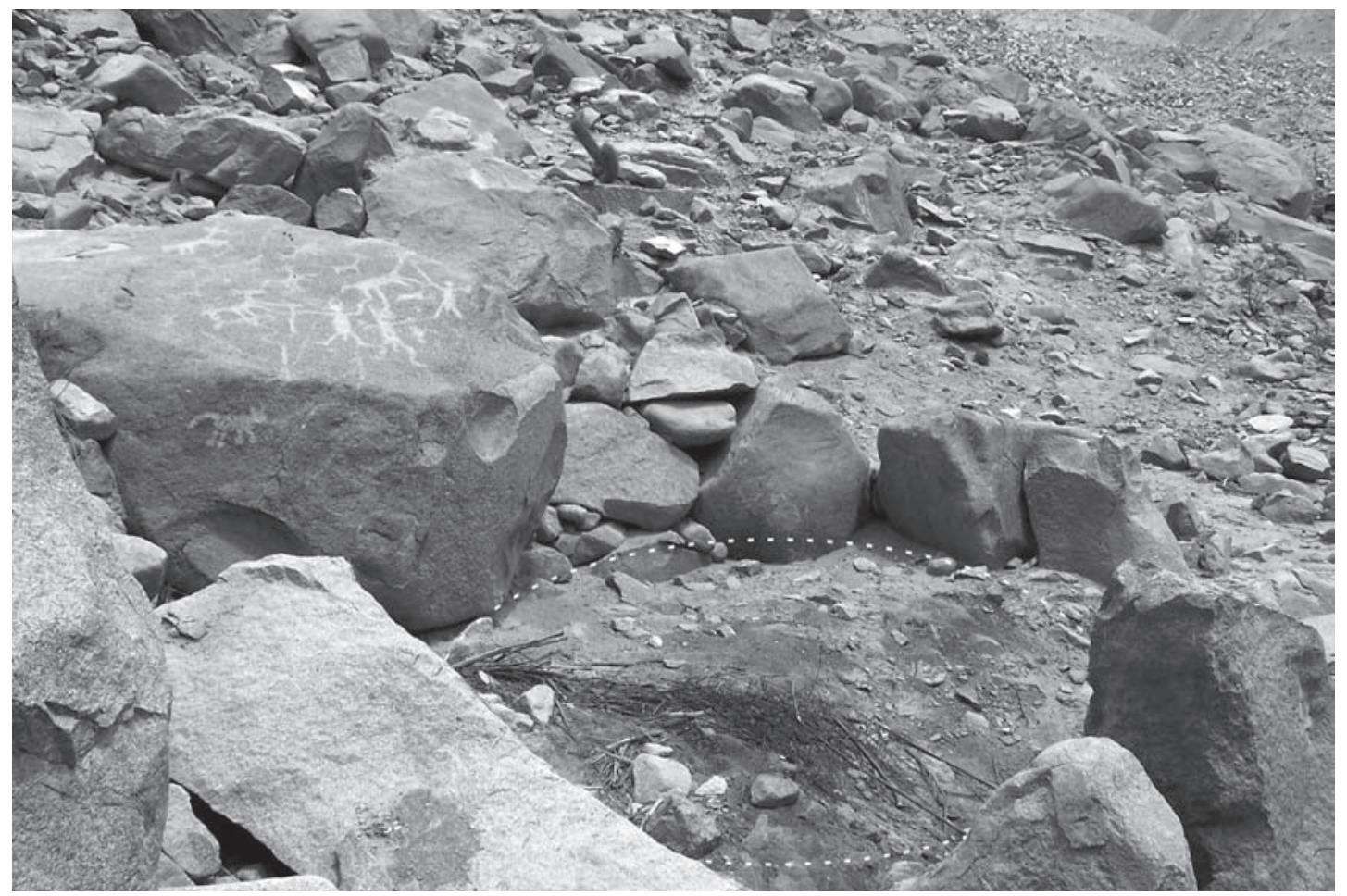

Figura 3. Asociación de una piedra grabada con una tumba circular (La Capilla, quebrada de Palka).

Association of an engraved rock with a circular grave (La Capilla, quebrada de Palka). 


\section{La Composición de las Superficies Grabadas}

La composición de las superficies grabadas constituye un buen marcador de las evoluciones ocurridas entre el periodo Formativo y el fin de la época prehispánica.

Las figuras tempranas ocupan generalmente la parte central, si no la totalidad, de la cara grabada (Tradición A). Se encuentran, además, frecuentemente aisladas, sin muchos signos en su alrededor. A veces, tal como en el caso de las serpientes de Alto de las Guitarras, la representación está constituida por la piedra en sí, con agregación de unos trazos que figuran ciertas particularidades anatómicas. Las figuras representadas corresponden, generalmente, a seres sobrenaturales antropomorfos, con a menudo rasgos felinos (Figura 4) y en diversas actitudes: con las manos alzadas o cruzadas, en actitudes de enfrentamiento, tocando música, o volando, entre otros. Se reconoce, también, la representación de la trilogía animal clásica del Formativo: felinos-aves rapaces-serpientes, sea bajo una forma naturalista o como "monstruos" formados por la combinación de varios elementos (serpiente con cabeza felina o de ave...). Existen igualmente unos escasos signos,

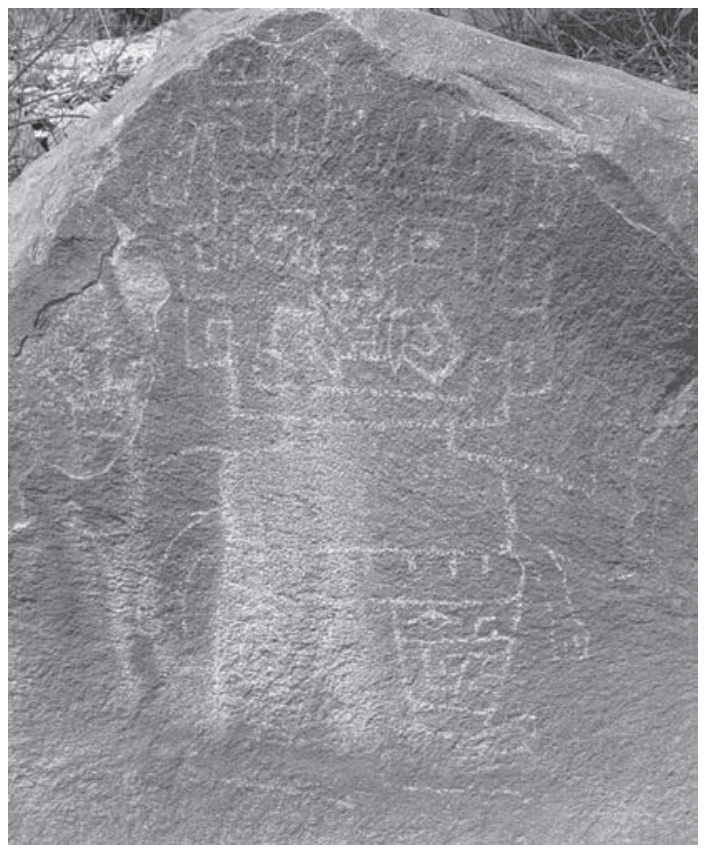

Figura 4. Ser sobrenatural con rasgos felinos grabado en Palamenco (río Lacramarca).

Engraved supernatural being with feline features from Palamenco (Lacramarca River). tales como el ojo excéntrico, la boca dentada y la cruz propios de esa época.

En la época siguiente, en la segunda Tradición $\mathrm{B}$, coexisten varios tipos de composición. La más característica corresponde a grandes superficies grabadas cubiertas de figuras de pequeñas dimensiones, yuxtapuestas o unidas por trazos grabados (Figura 5). Tales motivos, de difícil interpretación, se encuentran mayoritariamente en los sitios de la costa norte y costa central. Sin embargo, en los mismos yacimientos, ciertas categorías de figuras, tales como las representaciones animales o las cabezas y seres antropo-zoomorfos, se encuentran más frecuentemente aisladas u ocupan la parte central de la cara grabada. Pequeños motivos se hallan a menudo grabados en el entorno de la figura principal. En esta tradición, se encuentran también asociaciones repetidas de figuras que parecen formar referencias a mitos o historias particulares. Se nota el predominio de los signos (soles, cruces, cúpulas, ruedas...), además de una representación muy diversa de la pequeña fauna local: aves, serpientes, lagartijas, pescados, insectos y pequeños mamíferos. La referencia a los felinos es escasa en esta tradición. Los seres humanos se encuentran a menudo representados de frente y con los brazos abiertos. En la parte norteña y nororiental del territorio peruano, así como en el sur del Ecuador, existen representaciones comparables aunque un poco distintas y menos diversas. Predominan allí, los seres y cabezas antropomorfas, las serpientes, las espirales y las representaciones de pies y manos. Los petroglifos se ubican de forma yuxtapuesta sobre piedras de gran tamaño. Las figuras de aves, felinos, insectos y mamíferos están ausentes o son muy poco frecuentes.

La tercera gran tradición C, sureña, se caracteriza por nuevos temas, que permiten también establecer ciertas subdivisiones. En Toro Muerto se desarrolló una tradición particular, bastante estereotipada tanto en las figuras grabadas como en su composición. Predominan las representaciones de diversas especies de aves, de serpientes, de felinos vistos de perfil, jugando o peleando, de antropomorfos en actitud de danzar, así como algunos signos repetidos: zigzags, rayas verticales y cúpulas. Sobre una mayoría de rocas, el grabado parece haber seguido un esquema repetido, basado en la asociación codificada de rayas verticales, zigzags, cúpulas con figuras humanas (danzantes) y/o animales (Figura 6). Este tipo de composición es propia del sitio de Toro Muerto, 


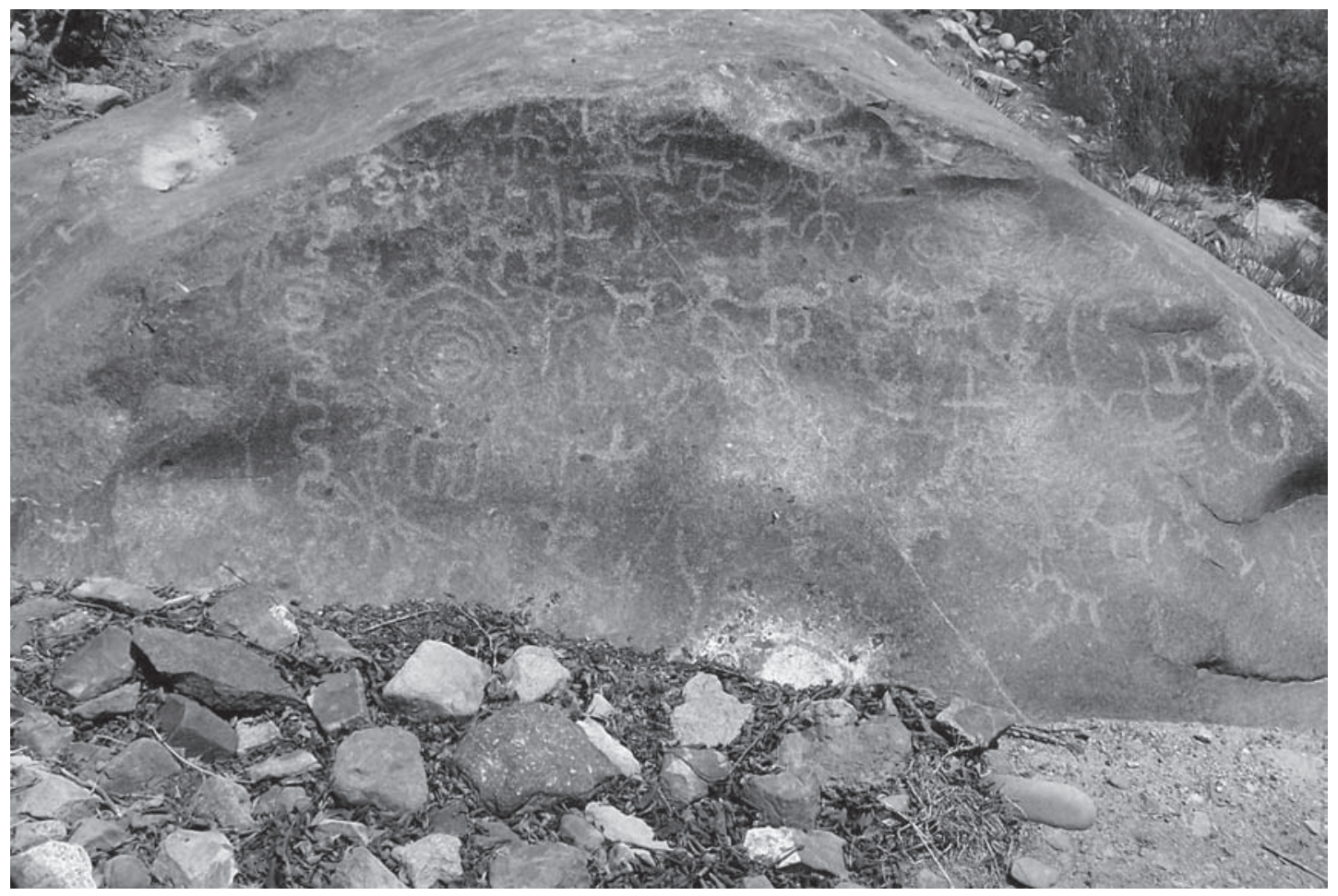

Figura 5. Piedra grabada en Cochineros (río Mala).

Engraved rock from Cochineros (Mala River).

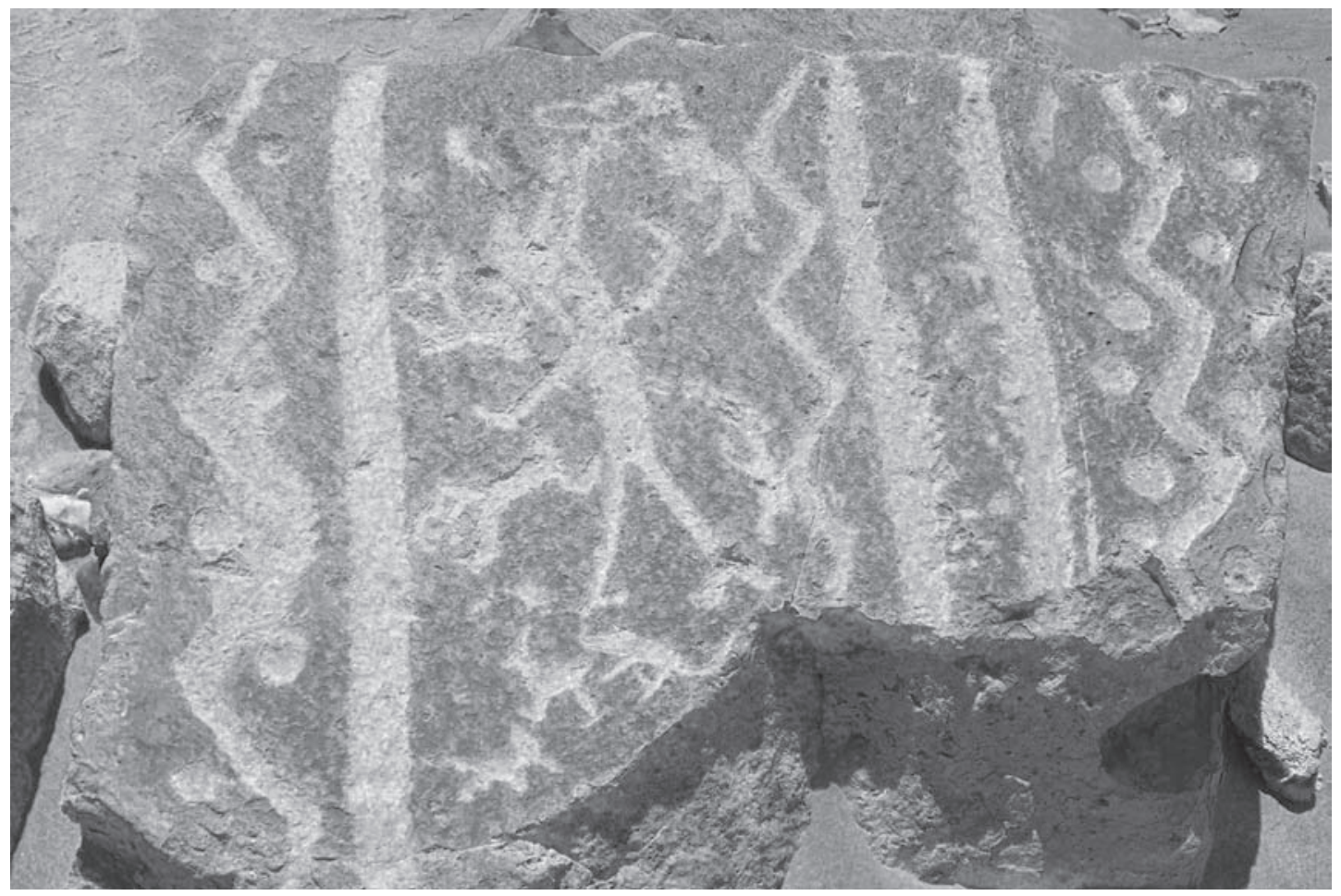

Figura 6. Piedra grabada en Toro Muerto (río Majes).

Engraved rock from Toro Muerto (Majes River). 
pero una iconografía semejante (con predominio de representaciones de felinos) se observa en otros sitios de los valles de Palpa (Chichictara), Majes (Messana) y Sihuas (Quilcapampa). Más al sur, en los departamentos de Moquegua (Locumba) y Tacna (Miculla, Quebrada de Palka), predominan las figuras humanas con tocados de cabeza, en escenas de caza, enfrentamiento o pastoreo; así como las representaciones de cuadrúpedos, serpientes y aves. Las figuras de felinos vuelven a ser escasas en esta región.

\section{Las Relaciones Culturales}

Los petroglifos tempranos (tradición A) que se relacionan claramente con la iconografía religiosa del periodo Formativo corresponden, por lo general, a figuras que no se repiten y que muestran, en cada sitio, una cierta singularidad. Las manifestaciones posteriores se caracterizan al contrario por la presencia de motivos o asociaciones repetidas, sea en el mismo sitio, sea en sitios alejados en el espacio y, a veces, en el tiempo. El tema es demasiado amplio para ser detallado aquí. Podemos, sin embargo, ilustrar nuestro planteamiento con ejemplos de diversos tipos de relaciones.

La presencia de motivos semejantes sobre un territorio más o menos amplio permite definir tradiciones locales, que suelen estar ligadas a complejos culturales identificados por la arqueología. Ya señalamos la existencia de tal corpus iconográfico (espirales, cabezas y seres antropomorfos, serpientes bicéfalas) en el extremo norte y nororiente de Perú y sur oriente de Ecuador (tradición B norteña). En la tradición de la costa norte y central (tradición B sureña) se puede también reconocer un conjunto de motivos grabados comparables a los observados en la iconografía de las culturas Mochica y Chimú (Yonán, Queneto, Quebrada de la Guitarra) y otro grupo más sureño, y probablemente un poco más temprano (Checta, Huancor), con iconografía particular: motivos complejos y solares, pequeños animales y seres sobrenaturales. Más al sur, los sitios aparentados a la cultura Nazca (Chichictara) tienen elementos comunes (felinos, aves, serpientes, músicos) con los sitios del Majes (Toro Muerto) y Sihuas (Quilcapampa; Tradición C, versión norteña), siendo probablemente un poco más tardíos, durante el Horizonte Medio o Intermedio Tardío. Un último grupo (Tradición $C$, versión sureña), con hombres con tocados de cabeza y manadas de camélidos, se encuentra principalmente en el extremo sur (Locumba, Miculla) y se relaciona con los períodos Intermedio Tardío e Inca y parte del arte grabado en el extremo norte chileno (sitio El Rosario-Arica, Valenzuela et al. 2004). El periodo Incaico parece haber sido un momento importante de difusión del arte grabado, particularmente en las zonas serranas y selváticas, donde predomina, desde muy temprano, el arte pintado.

Otro tipo de asociación corresponde a figuras singulares o conjuntos de figuras que están presentes en diferentes regiones y diversas épocas. Es el caso de ciertas actitudes (brazos levantados, un brazo levantado, otro bajado, brazos abiertos; Guffroy 1999: Figuras 35-36), o particularidades (cuerpo rayado, tocado de cabeza, cabellos) de los seres antropomorfos, humanos o sobrenaturales. Pertenecen a esta misma categoría, la representación de temas y motivos comunes en las culturas andinas, tales como la serpiente bicéfala (Guffroy 1999: Figura 46) o la relación entre aves, serpientes y felinos.

Finalmente, podemos reconocer asociaciones más complejas que parecen reflejar historias particulares. Aunque las representaciones se hallan formalmente diversas, es posible encontrar una estructura comparable entre rocas grabadas de los sitios Alto de las Guitarras, Checta y Huancor, donde aparecen las figuraciones de un ave, una cruz o cabeza antropomorfa y de una probable serpiente o ser serpentiforme (Figura 7). Algunos motivos, tales como las aves pescadoras de Yonán (mito de Naymlap) y las cabezas emplumadas de Checta (mitos de Huarochiri) remiten muy probablemente a mitos conocidos.

\section{Las Rocas con Cúpulas, Pocitos y Copas}

La presencia de grandes rocas que tienen una cara plana o ligeramente inclinada, cavada con depresiones circulares, constituye otro elemento que permite poner en evidencia la existencia de importantes evoluciones, pero también de relaciones, entre los diferentes complejos. Estas depresiones presentan diferentes tipos, diferenciados por sus diámetros y profundidades: las cúpulas, de menor tamaño; los pocitos, con una profundidad superior a su diámetro, y las copas, de mayor circunferencia pero con reducida profundidad.

Las copas aparecen estrechamente ligadas a las manifestaciones arquitecturales del fin del Formativo, 


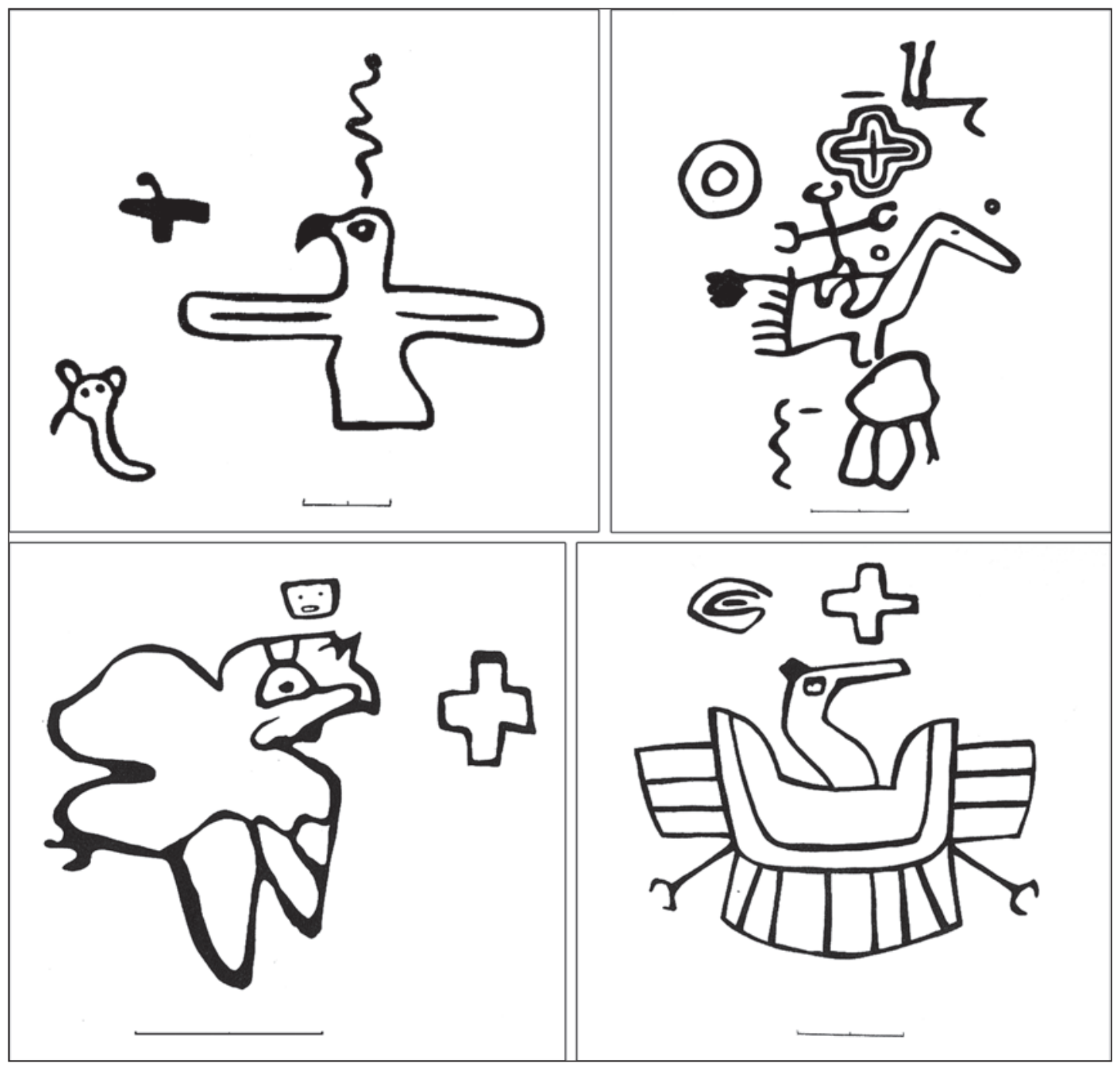

Figura 7. Asociación de figuras comparables, en Alto de las Guitarras, Checta y Huancor (según Núñez 1986). Association of comparable pictures from Alto de las Guitarras, Checta and Huancor (after Núñez 1986).

como en los casos del altar de Cho'qe Chinchay en Chavin de Huantar o los altares de Udima. Aparecen también en sitios sin arquitectura, al lado de otras manifestaciones rupestres (petroglifos, estelas), en sitios como Chiñama (río Motupe), Cerro La Cal (río La Leche; Alva y Ventura 2007) y Cerro Bolo (río Chinchipe) en el nororiente (Gamonal 2006). Representan probablemente una fase temprana del desarrollo de estas manifestaciones.

Las rocas planas con pocitos pueden encontrarse aisladas o dentro de sitios con petroglifos. Su área de repartición abarca desde Yonán (Figura 8), en el valle del Jequetepeque, hasta el Oriente ecuatoriano (Misagualli), incluyendo el departamento de Piura
(Paimas, Cerro Cuchalín), la provincia de Loja (Sosoranga, Sacapianga), en Ecuador (González 2004), y aquella de Jaen, en Perú (Chontali, Pikijaca; Gamonal 2006). Los pocitos profundos casi nunca aparecen en los yacimientos de la costa central y sur. Su número es variable, pero por lo general reducido (entre uno y siete). Estas piedras se encuentran a menudo en la parte alta de los cerros o cercana a los ríos. Parecen representar una evolución de la tradición anterior.

Grandes rocas planas cubiertas con cúpulas de diámetro inferior a $5 \mathrm{~cm}$ fueron grabadas en un gran número de sitios de la costa norte y central, tales como Udima, Sechin, Huaricanga, Caral, 


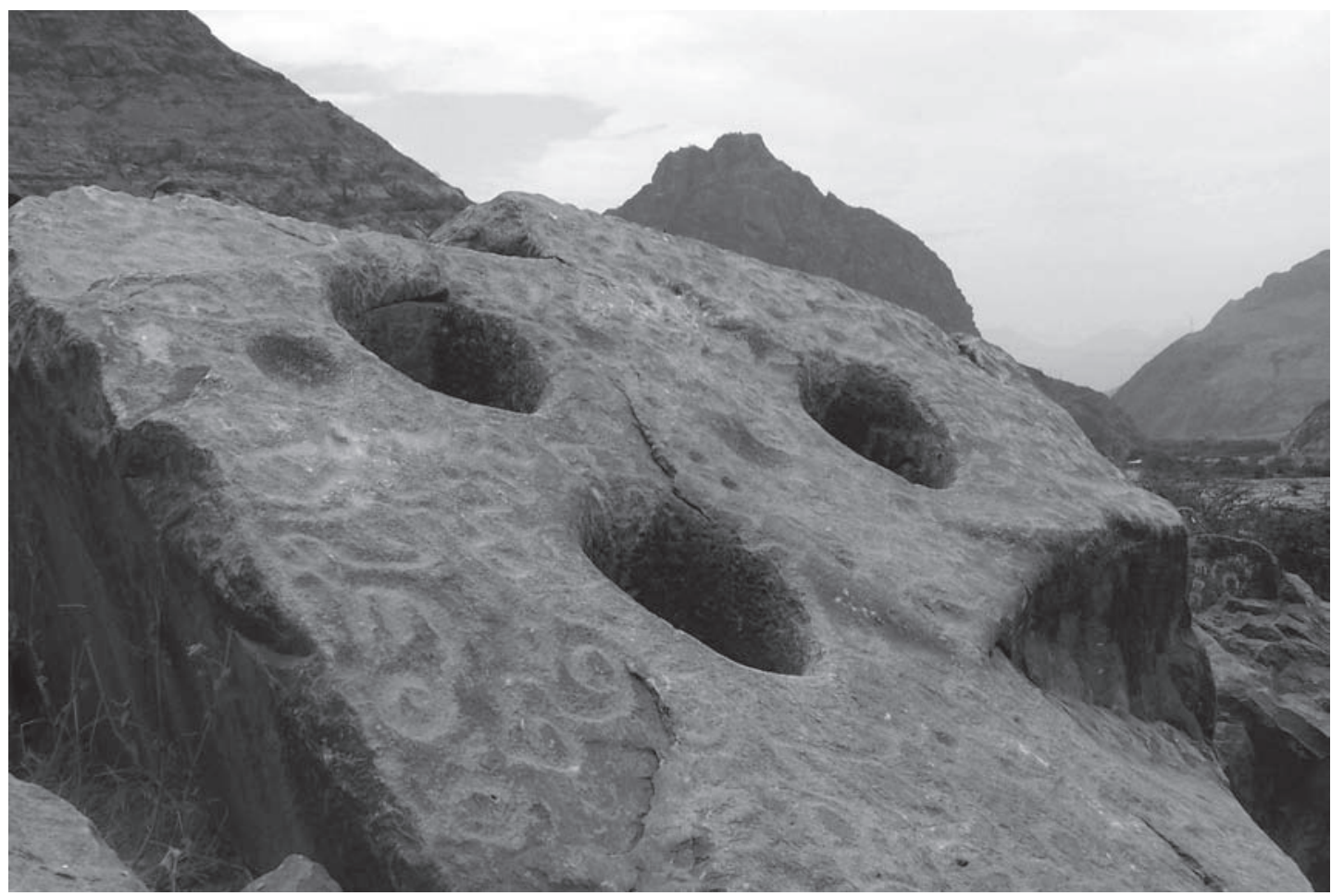

Figura 8. Roca con pocitos en Yonán (río Jequetepeque).

Rock with circular depressions from Yonán (Jequetepeque River).

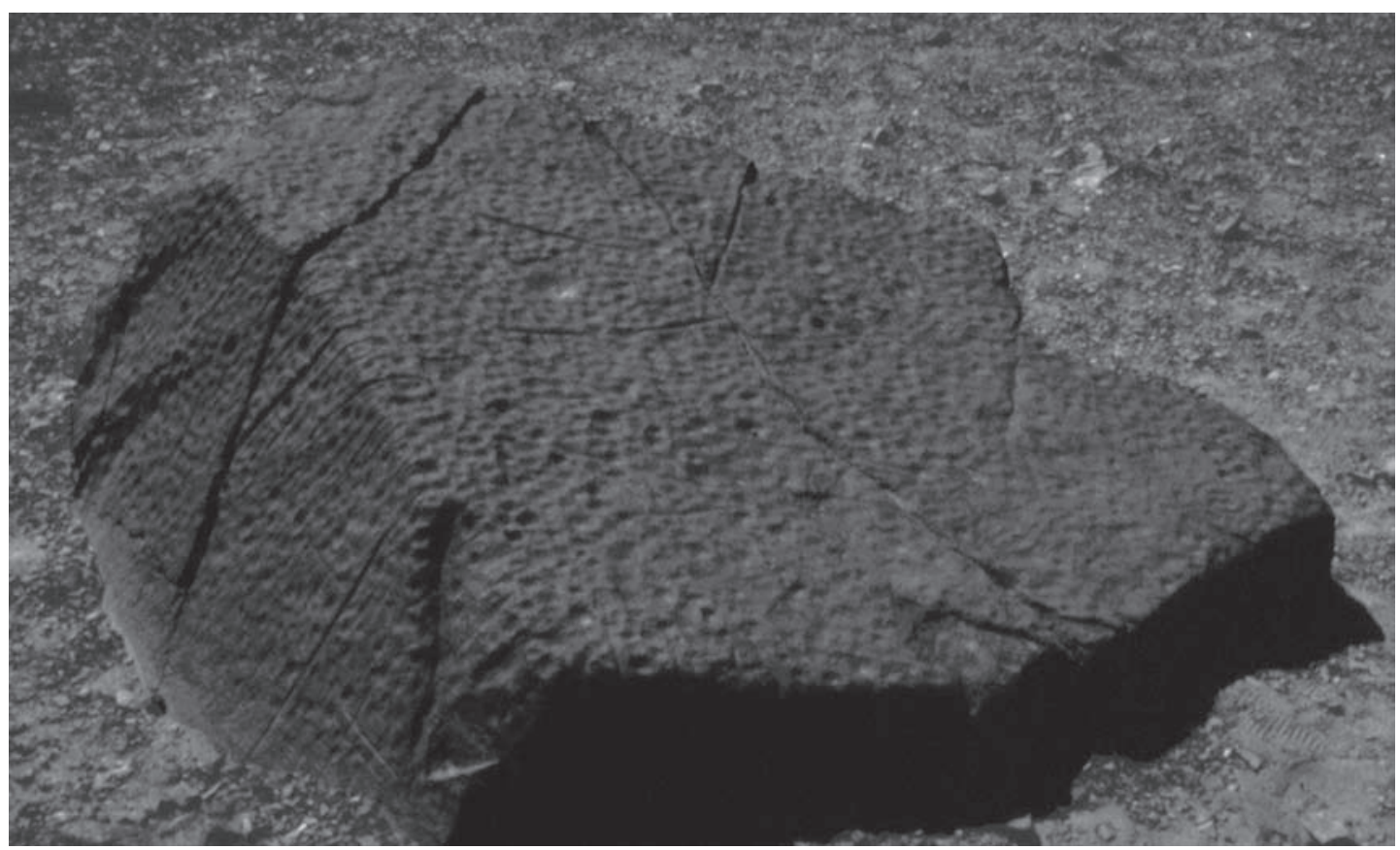

Figura 9. Piedra cubierta por cúpulas y ranuras de pulimento en Checta (río Chillón). Rock covered with cupules and polished grooves from Checta (Chillón River). 
Checta (Figura 9), Antapucro, Huancor y en el sur en sitios como Messana, Locumba y Quilcampa. Por sus características y emplazamientos parecen haber jugado un papel importante en los rituales y prácticas sociales realizadas en estos sitios (Guffroy 1999, 2007). En Checta, donde existe también una versión portátil, realizada sobre un canto rodado, tienen una función importante en la delimitación del área grabada y en su organización interna.

Las cúpulas entran también en la composición de paneles, bajo formas específicas: alineadas sobre las aristas de los bloques, en la Tradición B norteña; encerrada dentro de motivos geométricos (escudo, rueda) o animales (serpientes, aves, felinos), en la costa norte y central; y, en asociación con zigzag y rayas en Toro Muerto.

Se han dado interpretaciones funcionales diversas, y muy especulativas, de estas manifestaciones. Las copas fueron a menudo definidas como espejos, posiblemente relacionados con observaciones cosmológicas. Los pocitos fueron más frecuentemente relacionados con funciones de molienda, cultos al agua y ritos de fertilidad (Alva y Ventura 2007; Polia 1995). Las rocas con cúpulas, que a veces como en Checta llevan también ranuras de pulimento (Figura 9), suelen asociarse a prácticas de sacrificios y/o de adivinación. Pueden también haber significado una referencia al cosmos o cumplir con una función recordatoria, en algunos sitios tempranos. Sería importante determinar si la acumulación de cúpulas resulta de una actividad repetida en un largo lapso de tiempo o si representa una obra en sí, realizada de esa manera para tener su apariencia final. Sobre una de las dos piedras de Checta queda una zona poco cubierta de cúpulas que podría ser interpretada como signo de un trabajo todavía en proceso. Por otro lado, aunque tienen diámetros diferentes, todas las cúpulas enseñan un mismo aspecto formal que no sugiere el acto de diferentes productores. Las numerosas ranuras de pulimento que cortan las aristas y las paredes verticales de la roca representan tal vez más claramente una acumulación de actos (probablemente afilamiento de herramientas de piedra) y repetidos durante bastante tiempo. En el caso de los pocitos y copas, su realización parece corresponder más bien a un acto único, tal como lo sugiere la presencia de petroglifos en la parte superior de uno de los pocitos de Yonán.

Cual sea la veracidad de estas interpretaciones, la distribución de los diversos tipos de depresiones parece indicar una cierta evolución en los usos, así como singularidades culturales regionales. Su asociación variable con petroglifos, estelas, monumentos, o aisladas, las grandes rocas parece indicar que las piedras con depresiones tuvieron una significación particular, jugando un papel importante en los rituales desarrollados en los sitios donde aparecen. Existe probablemente una cierta correspondencia ideológica y ritual entre las piedras con cúpulas y aquellas con pocitos, probablemente contemporáneas, durante el Intermedio Temprano, pero con áreas de repartición diferentes. Diversos tipos de piedras labradas más tardíamente, particularmente durante el periodo Incaico, tal como la piedra de Saihuite o el adoratorio de Kenko y las piedras y adobes huaqueados de Pachacamac, podrían representar evoluciones tardías de esta misma tradición.

Por último, hay que señalar la existencia, en varios sitios de la versión sureña de Tradición B (Yonán, Checta, Huancor), de un punteado superficial voluntario de ciertas figuras o partes de rocas. En Checta, estas percusiones repetidas conciernen, por ejemplo, una roca grabada con dos representaciones de un mismo insecto (un saltamontes y su larva), posible objeto de un rito de execración.

\section{Correlaciones Finales... A Modo de Recapitulación}

La correlación de los datos anteriores permite confirmar la existencia de varios complejos (Figura 10) que representan no solamente etapas cronológicas y tradiciones culturales diversas, sino también probables variaciones en las prácticas sociales y las actividades ligadas a la presencia de piedras grabadas. Estas prácticas son difíciles de interpretar y más todavía de reconstruir. Podemos, sin embargo, adelantar algunas hipótesis basadas sobre las características generales de cada complejo.

En la época Formativa (Tradición A) predominan los pequeños sitios, más o menos apartados, y las piedras aisladas. Aunque existen algunas excepciones (tal como en Alto de las Guitarras) (Campana, comentario personal), el numero de petroglifos grabados sobre cada piedra es, por lo general, reducido. Algunas figuras, tal como "el prisionero del tiempo" en Alto de las Guitarras (Campana 2007), muestran gran complejidad, tanto en su concepción como en su ejecución. Las figuras grabadas presentan una gran semejanza con la iconografía de la época. Se integran, por lo tanto, en un corpus iconográfico representado sobre varios otros soportes (textiles, 


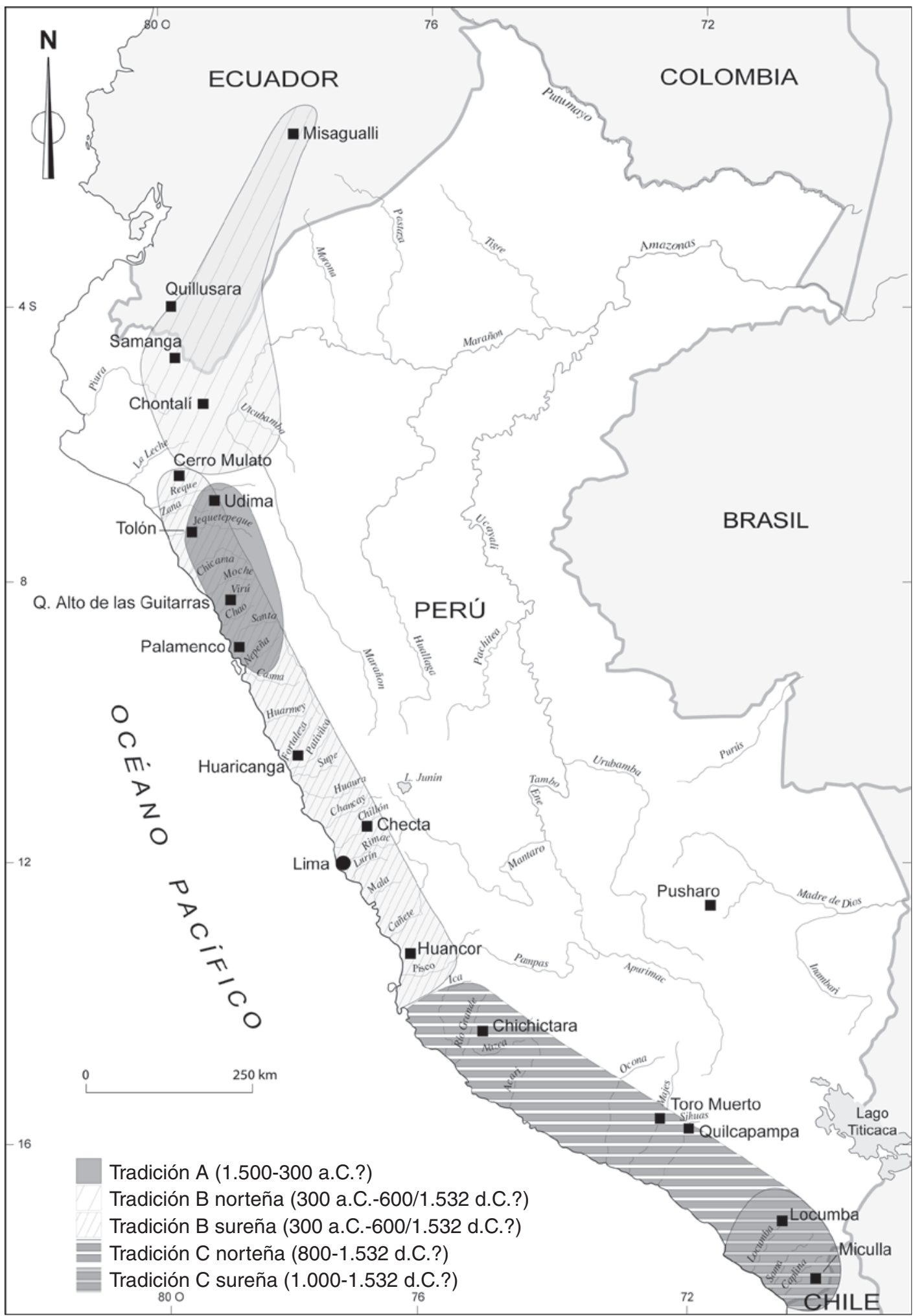

Figura 10. Áreas de repartición de algunas grandes tradiciones de petroglifos. Areas of repartition of some principal traditions of engraved rock art. 
ceramios, orfebrería, escultura, pinturas), como una figura claramente ligada al universo mineral y al entorno natural. Pudo existir una cierta veneración de la piedra, santificada con la representación pétrea del ser figurado. Existe, por lo general, una cierta facilidad de acercamiento y de tocado de la roca grabada, que se inscribe en su paisaje natural y cultural. El acto de grabar, que ocupa un corto espacio de tiempo en la vida del sitio, puede haber sido público o escondido; y cada figura fue, generalmente, realizada con bastante cuidado y con una cierta calidad artística que podría resultar de la intervención de artesanos formados en otros campos artísticos. Lo importante radica en la figura plasmada y en la relación del público hacia dicha figura por lo general bien conocida por hallarse representada y difundida sobre otros soportes materiales. En la misma época aparecen, dentro de algunos centros ceremoniales, grandes piedras planas con depresiones circulares no muy profundas, posiblemente relacionadas con el mundo cósmico y diversos rituales (astrología, sacrificios, adivinación).

Las tradiciones posteriores comparten ciertos puntos comunes, que las diferencian de la primera. Las figuras grabadas en una misma piedra suelen ser numerosas, siendo muy probablemente el producto de actos ocurridos durante cierto lapso de tiempo. Se observa recurrentemente la presencia de agregados de otras representaciones alrededor de una figura central. En algunos sitios, el acto de grabar viene a ser más común y repetido, probablemente realizado sin mucho protocolo, dentro de un arreglo general que puede haber sido codificado y planificado. Numerosos grabados corresponden a motivos simples que se repiten: rayas, círculos, zigzags, cruces, cúpulas. Las figuras naturalistas, que representan un porcentaje menor del universo total de representaciones son, en su mayoría, propias a la iconografía rupestre, hallándose escasamente en otros soportes materiales como textiles, murales o cerámicas. Los petroglifos no siempre fueron realizados con cuidado, un gran número siendo muy sencilla y rápidamente grabado. Una cierta cantidad de personas estuvieron probablemente implicadas en la materialización de los petroglifos; el acto de grabar teniendo una significación en sí. Los sitios con petroglifos pudieron haber tenido un rol importante en la vida social y religiosa de ciertos grupos de población.

Pero estas tradiciones presentan también diferencias marcadas que probablemente testimonian de la existencia de funciones y usos diferentes.
En la Tradición B, versión norteña, el relativo aislamiento de las piedras, sobre un territorio a menudo vasto o a lo largo de posibles caminos, deja entrever una función territorial y una inscripción difusa en el paisaje y, por lo general, sin agrupación particular. Aunque, la cantidad de petroglifos puede ser importante en unos sitios como es el caso de Samanga, suele ser más frecuentemente reducida, y con cierta repetición de los motivos.

Los petroglifos hacen parte del paisaje cultural, al lado de otros elementos rituales tales como las estelas o las piedras con pocitos o copitas. Los sitios con piedras grabadas, que suelen encontrarse apartados de las zonas habitacionales, no parecen haber sido lugares de grandes reuniones u objetos de prácticas rituales muy complejas.

Dentro de la versión sureña de la Tradición $\mathrm{B}$, los sitios de pendiente o de quebrada (Cerro Mulato, Yonán, Huancor, Chichictara) presentan figuras grabadas visibles desde lejos, pero de acceso a menudo difícil. Estos yacimientos tienen frecuentemente un aspecto teatral, con sectores que pudieron estar reservados para ciertos tipos de actos u actividades. En los sitios de planicie (Alto de las Guitarras, Palamenco, Checta) existe la posibilidad de desarrollar actividades variadas, como danza, ruego, ofrenda, peregrinación, adivinación, sacrificio., sobre un espacio más o menos vasto, con la participación de una importante concurrencia. El espacio ritual suele ser delimitado y organizado según criterios discretos.

Los sitios de la costa norte y central estuvieron muy probablemente ligados estrechamente a ritos agrarios y muy posiblemente, en varios sectores, con el cultivo, la cosecha y la distribución de la coca. Los petroglifos se refieren a la mitología y la cosmología andina, plasmada de manera sencilla sin muchos estereotipos, pero con figuras a menudo difíciles de interpretar. El tema de los felinos es poco tratado, las cabezas antropomorfas o antropo-zoomorfas siendo mucho más frecuentes. Algunas piedras, grabadas en sitios monumentales más tempranos, podrían haber tenido una función recordatoria. Las figuras están a menudo plasmadas sin maestría artística particular, lo que podría indicar una cierta popularización del acto de grabar.

En la Tradición C (tanto en el departamento de Arequipa, como en Moquegua y Tacna) parece haber existido una estrecha relación con las prácticas funerarias y las probables rutas de 
intercambio (Núñez 1976; Gordillo 1996). El sitio de Toro Muerto se singulariza por la invención de un código y de una organización estereotipada de los paneles basada en la reproducción de unos motivos básicos de carácter simbólico, fácilmente identificables. Entre los motivos destacan las rayas, zigzags, cúpulas, antropomorfos, felinos, aves. $\mathrm{El}$ acto de grabar consiste principalmente en la repetición de una iconografía simbólica, y no en la invención o creación de nuevas figuras. La trilogía felinos-serpientes-aves rapaces predomina, con los danzantes, en el universo iconográfico. El hecho de regresar delante de las piedras ya grabadas puede haber tenido una menor importancia. Tampoco se vislumbra claramente la existencia de sectores con funciones rituales particulares. Los petroglifos fueron generalmente grabados con bastante cuidado y demuestran una buena maestría artística, que parece reflejar actos de individuos acostumbrados o especializados.
La versión sureña de la Tradición C (Moquegua, Tacna) reanuda con una cierta innovación, diversas figuras complejas y piedras con cúpulas, con rasgos semejantes a la Tradición B, versión sureña.

Obviamente, hay sitios con rocas grabadas que no entran claramente en la crono-tipología expuesta. Sus eventuales relaciones con las tradiciones descritas y/o la existencia de otros complejos quedan por aclarar. La confirmación de las correlaciones propuestas, así como de las hipótesis respecto de los usos y funciones asociadas, se encuentran a la espera del desarrollo de nuevos estudios de terreno y análisis detallados de contextos aún demasiados escasos en el Perú.

Agradecimientos: Quisiera agradecer a los evaluadores C. Campana, K. Makosk y M. Strecker, por sus observaciones pertinentes que me ayudaron a mejorar el texto así como a M. Sepúlveda por su apoyo en la preparación del presente artículo.

\section{Referencias Citadas}

Abanto, J. y M. García-Godos

2004 Los petroglifos formativos de Cerro Cantería, Lima. Primer encuentro peruano de arte rupestre. Arkeos, Revista Electrónica de Arqueología. PUCP, Lima.

Alva I. y R. Ventura

2007 Los petroglifos de Cerro La Cal: un santuario formativo en el valle de Chancay (Lambayeque). Actas del Primer Simposio Nacional de Arte Rupestre, Cusco 2004, editado por R. Hostnig, M. Strecker y J. Guffroy, pp 19-35. IFEAIRD-Embajada de Alemania, Lima.

Bray, $\mathrm{T}$.

2001 Current researchs in andean archaeology; Pimampiro project. Andean Past 6:369-372.

Bustinza, J.

1991 Arte Rupestre en el Valle de Salcedo. Una Introducción a su Estudio. Visión mundial internacional, Puno.

Campana, C.

2007 El prisionero del tiempo, un petroglifo de Alto de las Guitarras. Actas del Primer Simposio Nacional de Arte Rupestre, Cusco 2004, editado por R. Hostnig, M. Strecker y J. Guffroy, pp. 37-67. IFEA-IRD-Embajada de Alemania, Lima.

Del Carpio, M., M. Mac Kay M. y R. Santa Cruz

2001 Poro Poro: religión y agua en el formativo de la sierra norte peruana. Revista Arqueológicas 25:95-116. Museo Nacional de Arqueología, Antropología e Historia del Perú.

Duviols, P.

1971 La lutte contre les religions autochtones dans le Pérou colonial, l'extirpation de l'idolâtrie entre 1538 et 1660. IFEA, Lima.

Gamonal, U.

$2006 \mathrm{El}$ arte rupestre en el nororiente peruano. Facetas 55:13-23.
González, D.

2004 El Arte Rupestre de Loja. Universidad Técnica Particular de Loja, Loja.

Gordillo, J.

1996 Miculla, Petroglifos: la Magia de los Signos. Ediciones EPF, Tacna.

Guffroy, J.

1999 El Arte Rupestre del Antiguo Perú. IFEA-IRD, Lima. 2003 New researches and discoveries in Peruvian rock art studies. En Rock Art Studies: News of the World 2, editado por P.G. Bahn y R. Fossati, pp. 221-226. Oxbow, Oxford.

2007 Reflexiones acerca del funcionamiento y de las finalidades de los sitios con piedras grabadas. Actas del Primer Simposio Nacional de Arte Rupestre, Cusco 2004, editado por R. Hostnig, M. Strecker y J. Guffroy pp. 117-131. IFEA-IRD-Embajada de Alemania, Lima.

Hostnig, R.

2003 Arte Rupestre del Perú, Inventario Nacional. CONCYTEC, Lima.

Linares, E.

1960 Notas sobre los petroglifos de Toro Muerto. En Antiguo Perú, Espacio y Tiempo, pp 297-301. Editorial J. Meria Baca, Lima.

Núñez, L.

1976 Geoglifos y tráfico de caravanas en el desierto chileno. Anales de la Universidad del Norte 10:147-201. Antofagasta.

Núñez Jiménez, A.

1986 Petroglifos del Perú. Panorama Mundial del Arte Rupestre. Ministerio de Cultura, Editorial Científico Técnica, La Habana. 
Pimentel, V.

1986 Petroglifos en el valle medio y bajo de Jequetepeque, norte del Perú. Materialien zur Allgemenein und Vergleichenden Archaeologie; bd 31. München.

Polia, M.

1995 Los Guayacundos Ayahuacas: Una Arqueología Desconocida. Concejo Municipal de Ayabaca. Fondo Editorial Pontificia Universidad Católica del Perú, Lima. Shady, R.

2005 La Civilización de Caral-Supe: 5000 años de Identidad en el Perú. INC, Lima.
Valcarcel, L.E.

1926 Petroglifos de la Convención. Revista Universitaria XVI (51):4-14.

Valenzuela, D., C. Santoro y A. Romero

2004 Arte rupestre en asentamientos del Período Tardío en los valles de Lluta y Azapa, norte de Chile. Chungara Revista de Antropología Chilena 36:421-437.

van Hoek, M.

2005 Toro Muerto, Perú. Posibles alteraciones prehistóricas en detalles de petroglifos. http://rupestreweb2.tripod.com/ toromuerto.html. 